\title{
Excitation and propagation of surface plasmon polaritons on a non-structured surface with a permittivity gradient
}

\author{
Xi Wang ${ }^{1}$, Yang Deng ${ }^{1}$, Qitong $\mathrm{Li}^{1}$, Yijing Huang ${ }^{1}$, Zilun Gong ${ }^{1,2}$, Kyle B Tom ${ }^{1,3}$ and Jie Yao ${ }^{1,3}$
}

Accompanied by the rise of plasmonic materials beyond those based on noble metals and the development of advanced materials processing techniques, it is important to understand the plasmonic behavior of materials with large-scale inhomogeneity (such as gradient permittivity materials) because they cannot be modeled simply as scatterers. In this paper, we theoretically analyze the excitation and propagation of surface plasmon polaritons (SPPs) on a planar interface between a homogeneous dielectric and a material with a gradient of negative permittivity. We demonstrate the following: (i) free-space propagating waves and surface waves can be coupled by a gradient negative-permittivity material and (ii) the coupling can be enhanced if the material permittivity variation is suitably designed. This theory is then verified by numerical simulations. A direct application of this theory, 'rainbow trapping', is also proposed, considering a realistic design based on doped indium antimonide. This theory may lead to various applications, such as ultracompact spectroscopy and dynamically controllable generation of SPPs.

Light: Science \& Applications (2016) 5, e16179; doi:10.1038/Isa.2016.179; published online 2 December 2016

Keywords: gradient negative permittivity; non-structured surface; rainbow trapping; surface plasmonics

\section{INTRODUCTION}

Surface plasmon polaritons (SPPs) are bound electromagnetic waves propagating at a dielectric-metal interface with exponential field decay normal to the interface ${ }^{1}$. Because of SPPs' capability of deep subwavelength confinement and enhancement of the electric field as well as high sensitivity to the permittivity of the surrounding media, SPPs have been used in numerous applications, including sensing $^{2,3}$, imaging ${ }^{4}$, subwavelength aperture transmission ${ }^{5,6}$, nanophoton detectors ${ }^{7,8}$, nanoscale optical trapping ${ }^{9,10}$ and optical nonlinearities ${ }^{11,12}$.

Extensive applications of SPPs demand convenient and controllable methods for coupling light in free space to SPPs. Beyond random surface roughness ${ }^{13}$ or fluctuations in an electron gas ${ }^{14}$, structured surfaces are typically used to compensate for the mismatched momentum of SPPs and free-space light. Efficient excitation of SPPs has been achieved with specially designed structures, such as metallic gratings $^{15}$ and nanoslits ${ }^{16}$.

With the rise of non-conventional plasmonic materials, such as transparent conductive oxides and heavily doped semiconductors ${ }^{17-24}$, it has become possible to precisely control the permittivity distribution, thereby enabling new approaches for the excitation of SPPs. Conventionally, small fluctuations of permittivity have been considered as perturbations $s^{14,25}$ or scatterers ${ }^{26}$. However, materials with large-scale inhomogeneity, such as gradient permittivity materials, cannot be modeled as simple scatterers. In this paper, we show theoretically that the excitation and propagation of SPPs can be realized at a non-structured planar interface between a homogeneous dielectric and a gradient negative-permittivity material (GNM). With the development of advanced fabrication techniques, such as semiconductor doping ${ }^{27}$, electrical gating ${ }^{23,24}$, optical pumping ${ }^{28,29}$ and metamaterials ${ }^{30}$, gradient permittivity materials can be readily obtained. Spatial gradients of permittivity can be achieved at various length scales, from deep subwavelength to the macroscopic scale. Excellent scalability and dynamic tunability of GNMs will lead to a new platform for plasmonics research and device applications. In this report, we also apply our theory to the realization of 'rainbow trapping' without surface patterning.

\section{MATERIALS AND METHODS}

We divide our analysis into two parts: (i) the SPP propagation on a GNM; and (ii) the coupling of a free-space wave to a GNM surface.

We start by considering a surface wave propagating along the $+x$ direction at the interface between two materials with distinct permittivities ( $\varepsilon_{\mathrm{m}}$ for $z<0$ and constant $\varepsilon_{\mathrm{d}}$ for $z>0$ ) as shown in Figure 1a. The $y$ direction is assumed to be homogeneous. For constant negative $\varepsilon_{\mathrm{m}}$, i.e., a homogeneous metal, an SPP wave has been explicitly derived as a solution of Maxwell's equations ${ }^{1}$. In the inhomogeneous scenario, i.e., spatial variation of $\varepsilon_{\mathrm{m}}$ along $x$, the wave equation must be modified.

${ }^{1}$ Department of Materials Science and Engineering, University of California, Berkeley, CA 94720, USA; ${ }^{2}$ Environmental Energy Technologies Division, Lawrence Berkeley National Laboratory, Berkeley, CA 94720, USA and ${ }^{3}$ Materials Sciences Division, Lawrence Berkeley National Laboratory, Berkeley, CA 94720, USA

Correspondence: J Yao, Email: yaojie@berkeley.edu

Received 28 February 2016; revised 6 June 2016; accepted 7 June 2016; accepted article preview online 8 June 2016 

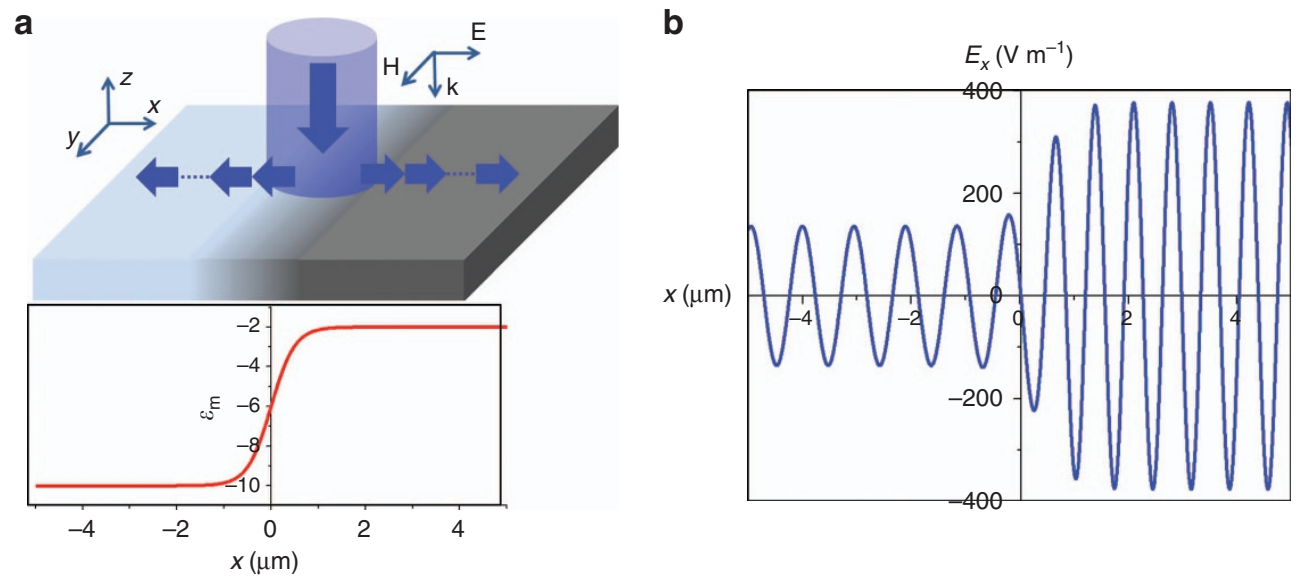

C

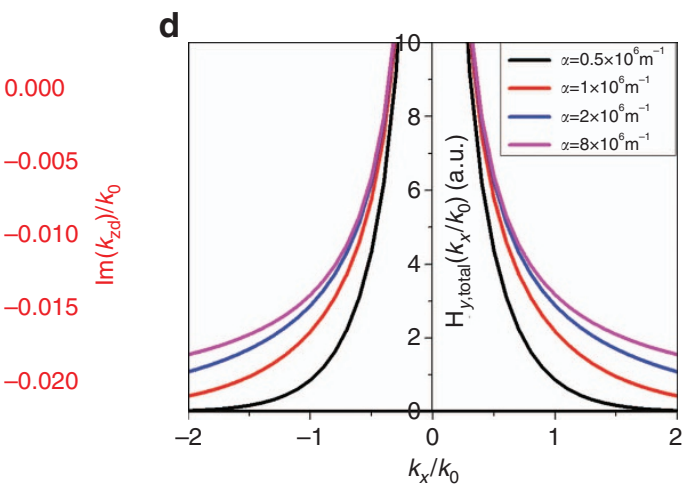

Figure 1 (a) Model schematic with an epsilon distribution. (b) $x$ component of the electric field distribution $\left(E_{x}\right.$ ) along the interface. (c) real (blue) and imaginary (red) parts of the decay factor $k_{z \mathrm{~d}}$ of the SPP in air. (d) spatial frequency spectra when a GNM with different values of $\alpha$ is illuminated by a TM-polarized plane wave.

We start from Maxwell's Equations, assuming a monochromatic transverse-magnetic (TM) polarized wave $\left(H_{x}=H_{z}=0, E_{y}=0\right)$ with angular frequency $\omega$. Because materials are homogeneous along the $z$ direction in each half space ( $z>0$ and $z<0$, respectively), we can write the magnetic field $H_{y}$ as:

$$
\left\{\begin{array}{l}
H_{y}=G(x) e^{-k_{z \mathrm{~d}}(x) z}, z>0, \operatorname{Re}\left(k_{z \mathrm{~d}}(x)\right)>0 \\
H_{y}=G(x) e^{k_{\mathrm{zm}}(x) z}, z<0, \operatorname{Re}\left(k_{z \mathrm{~m}}(x)\right)>0
\end{array}\right.
$$

where $G(x)=H_{y}(x, z=0)$ describes the magnetic field at the interface; and $k_{z \mathrm{~d}}$ and $k_{z \mathrm{~m}}$ are the decay factors of the SPP in air and in the GNM, respectively. By applying the continuity conditions of the tangential fields $\left(H_{y}\right.$ and $\left.E_{x}\right)$ on the interface $(z=0)$, we obtain:

$$
G^{\prime \prime}(x)+\frac{\varepsilon_{\mathrm{m}}^{\prime}(x) \varepsilon_{\mathrm{d}}^{2}}{\varepsilon_{\mathrm{m}}(x)\left[\varepsilon_{\mathrm{m}}^{2}(x)-\varepsilon_{\mathrm{d}}^{2}\right]} G^{\prime}(x)+k_{0}^{2} \frac{\varepsilon_{\mathrm{m}}(x) \varepsilon_{\mathrm{d}}}{\varepsilon_{\mathrm{m}}(x)+\varepsilon_{\mathrm{d}}} G(x)=0
$$

See Supplementary Material 1 for derivation details.

The above equation governs the behavior of the propagation of SPPs on the GNM. If $\varepsilon_{\mathrm{m}}$ is also a constant, then Equation (2) is simplified to the form of:

$$
G^{\prime \prime}(x)+k_{0}^{2} \frac{\varepsilon_{\mathrm{m}}(x) \varepsilon_{\mathrm{d}}}{\varepsilon_{\mathrm{m}}(x)+\varepsilon_{\mathrm{d}}} G(x)=0
$$

which is the standard form of an SPP. To study the behavior of an SPP between a homogeneous dielectric material and a GNM, we must solve Equations (1) and (2) to obtain $G(x)$ and then derive $H_{y}, E_{x}, E_{z}$ and $k_{z \mathrm{~d}}$. Without loss of generality, we set $\varepsilon_{\mathrm{d}}=1$, corresponding to vacuum, and $\varepsilon_{\mathrm{m}}(x)=\frac{\varepsilon_{\mathrm{m}, \text { min }}+\varepsilon_{\mathrm{m}, \text { max }}}{2}+\frac{\varepsilon_{\mathrm{m}, \text { min }}-\varepsilon_{m, \text { max }}}{2} \tanh (\alpha x)$, where $\varepsilon_{\mathrm{m}, \max }\left(\varepsilon_{\mathrm{m}, \min }\right)$ is the maximum (minimum) permittivity in the GNM. The form of $\varepsilon_{\mathrm{m}}$ following the tanh function is used to describe a realistic case when two semiconductors with different doping concentrations join together. The permittivity of a GNM is monotonically increasing from $\varepsilon_{\mathrm{m}, \min }$ to $\varepsilon_{\mathrm{m}, \max }$ along the $+x$ direction. ' $\alpha$ ' reflects the 'slope' of the tanh function in the region near $x=0$, i.e., the larger $\alpha$ is, the faster $\varepsilon_{\mathrm{m}}$ changes in the region near $x=0$. We define the region where $0.999 \varepsilon_{\mathrm{m}, \min }<\varepsilon_{\mathrm{m}}(x)<1.001 \varepsilon_{\mathrm{m} \text {,max }}$ as the 'transition area'; thus, the width of the transition area is $\sim 4 / \alpha$. Scatterers are preexcluded by setting such continuous permittivity functions over the whole interface to avoid their influence, similar to the procedure in the studies in refs 14 and 26.

We choose free-space wavelength $\lambda=1 \mu \mathrm{m}, \varepsilon_{\mathrm{m}, \max }=-2, \varepsilon_{\mathrm{m}, \min }=-10$ and $\alpha=2 \times 10^{6} \mathrm{~m}^{-1}$ as an example case. The permittivity distribution of the GNM is shown by the red curve in Figure 1a. To demonstrate an SPP wave propagating from $-\infty$ through the transition area towards $+\infty$, we set boundary conditions at $+\infty$ as $G(+\infty)=1$ and $G^{\prime}(+\infty)=i k_{0} \sqrt{\frac{\varepsilon_{\mathrm{d}} \varepsilon_{\mathrm{m}}(+\infty)}{\varepsilon_{\mathrm{d}}+\varepsilon_{\mathrm{m}}(+\infty)}}$, i.e., the transmitted SPP becomes a conventional SPP after the transition area. Obviously, when $\varepsilon_{\mathrm{m}}$ has no gradient, the solution of Equation (2) is $G(x)=e^{i k_{0}} \sqrt{\frac{\varepsilon_{\mathrm{m} \varepsilon_{\mathrm{d}}}}{\varepsilon_{\mathrm{m}}+\varepsilon_{\mathrm{d}}}}$, representing a normal SPP mode. When $\varepsilon_{\mathrm{m}}$ is not a constant, there is no analytical solution for Equation (2), and we must then solve it numerically.

The existence of SPP modes on the GNM surface can be verified from its $x$ component of electric field $\left(E_{x}\right)$ and the decay factor of the SPP in air $\left(k_{z \mathrm{~d}}\right)$. By plotting $E_{x}$ in Figure $1 \mathrm{~b}$, we notice that, outside the 
transition area, the electric field behaves the same as conventional SPPs. Consistent with our knowledge ${ }^{31}$, the amplitude of $E_{x}$ in the $\varepsilon_{\mathrm{m}}=-2$ region is larger than that in the $\varepsilon_{\mathrm{m}}=-10$ region, indicating different concentrations of fields near the surface in the two regions. In the transition area, however, a continuous gradient of phase and amplitude builds a non-abrupt connection between the two conventional SPPs.

The behavior of $k_{z \mathrm{~d}}$ shows the coupling of SPPs to free-space waves. We plot $k_{z \mathrm{~d}}$ from equation $k_{z \mathrm{~d}}^{2}(x)=-\left(k_{0}^{2} \varepsilon_{\mathrm{d}}+\frac{G^{\prime \prime}(x)}{G(x)}\right)$. The real part of $k_{z \mathrm{~d}}$ (the blue curve in Figure 1c) is as expected: it remains constant outside the transition area and increases continuously in the transition area. Meanwhile, the imaginary part of $k_{z \mathrm{~d}}$ (the red curve in Figure 1c) shows a very important behavior: although the imaginary part of $k_{z \mathrm{~d}}$ is zero everywhere outside the transition area, it becomes negative in the transition area. In our definition in Equation (1), imaginary $k_{z \mathrm{~d}}$ denotes a propagation component in the $z$ direction; thus, a negative value corresponds to a radiating wave towards the $+z$ direction (in air). This radiating component implies that SPPs and free-space waves are coupled in the transition area. This coupling enables the excitation of SPPs via illumination of the transition region. In contrast, in the absence of a GNM, i.e., $\varepsilon_{\mathrm{m}}$ is a constant negative value, the real part of $k_{z \mathrm{~d}}$ is a constant and the imaginary part of $k_{z \mathrm{~d}}$ remains zero everywhere.

In the following, we analyze the coupling mechanism of free-space light to an SPP on a GNM. We use a TM polarized plane wave to illuminate the GNM surface normally, under which circumstance the electric field is along the $x$ direction and the magnetic field is along the $y$ direction.

First, propagating wave illumination causes a single layer of electric dipoles to oscillate at the interface. Because the penetration length of the incident beam is much shorter than a wavelength in a negative permittivity material, this assumption is reasonable. Dipoles on the interface generate all spatial frequency components of the electromagnetic field. The radiating magnetic field of a single dipole located at $x^{\prime}$ on the surface of a GNM has the form below:

$H\left(r, t, x^{\prime}\right)=\frac{-\omega^{2}}{4 \pi c\left|r-x^{\prime}\right|} E_{0} e^{-i \omega t} \varepsilon_{0} \chi\left(x^{\prime}\right) \Delta z \mathrm{~d} x^{\prime} \times \sin (\theta) e^{i k \mid r-x^{\prime}} \mid e^{-i \omega t} \hat{\varphi}\left(r, x^{\prime}\right)$

where $\varepsilon=1+\chi$, and $\theta$ and $\varphi$ represent the elevation angle of the $x$ axis and the azimuth angle in the $y-z$ plane, respectively ${ }^{32}$.

We obtain the spatial spectra of the magnetic field radiated by dipoles across the whole interface by conducting a Fourier transformation ( $k_{y}$ is set to be zero because the electrons' response is uniform along the $y$ axis):

$H_{\text {total }}\left(k_{x}, k_{y}=0\right)$

$=\int \mathrm{d} x^{\prime} \int \frac{-\omega^{2}}{4 \pi c\left|r-x^{\prime}\right|} E_{0} e^{-i \omega t} \varepsilon_{0} \chi\left(x^{\prime}\right) \Delta z \times \sin (\theta) e^{i k\left|r-x^{\prime}\right|} e^{-i \omega t} \hat{\varphi}\left(r, x^{\prime}\right) e^{-i k_{x} x} \mathrm{~d} x \mathrm{~d} y$ $=A \int \chi\left(x^{\prime}\right) e^{-i k_{x} x^{\prime}} \mathrm{d} x^{\prime}$

where $A$ represents the spatial frequency spectra of the single dipole. According to Ref. 33, A should have the following form:

$$
H_{y}\left(k_{x}, z\right) \propto\left[p_{z} \frac{k_{x}}{k_{z}}-p_{x}\right] e^{i k_{z} z}, z>0
$$

With our restriction $p_{z}=0,|A|$ should be equivalent to a constant when $k_{x}$ changes. Thus, we can focus on the second factor:

$$
\int \chi\left(x^{\prime}\right) e^{-i k_{x} x^{\prime}} \mathrm{d} x^{\prime}
$$

Calculation of the integral as a function of $k_{x}$ gives the relative coefficient of the spatial frequency spectra shown in Figure 1d
(Supplementary Material 2). Here, we are focusing on amplitudes of excited magnetic field components with $\left|k_{x}\right|>k_{0}$, which are evanescent along the $z$ axis and recognized as surface wave components. These nonzero amplitudes indicate the existence of evanescent components, i.e., free space to SPP coupling occurs. In the absence of a GNM, i.e., the plane wave illuminating a homogeneous surface normally, the spatial frequency spectra is a delta function centered at $k_{x}=0$, indicating a purely reflected wave without any evanescent components. In addition, note that a higher rate of transition of the permittivity corresponds to the generation of stronger surface wave components. Because the plotted integral is an even function, the amplitudes of the excited surface wave components are equal in both the $-x$ and $+x$ directions.

The varying local permittivities in the transition region allow modes with matched $k_{x}$ to be coupled to positions, where $\quad k_{\mathrm{SPP}}(x)=k_{0} \sqrt{\frac{\varepsilon_{\mathrm{m}}(x) \varepsilon_{\mathrm{d}}}{\varepsilon_{\mathrm{m}}(x)+\varepsilon_{\mathrm{d}}}}$. $\quad$ Components satisfying $k_{0} \sqrt{\frac{\varepsilon_{\mathrm{m}, \min \varepsilon_{\mathrm{d}}}}{\varepsilon_{\mathrm{m}, \min }+\varepsilon_{\mathrm{d}}}} \leq k_{x} \leq k_{0} \sqrt{\frac{\varepsilon_{\mathrm{m}, \max } \varepsilon_{\mathrm{d}}}{\varepsilon_{\mathrm{m}, \max }+\varepsilon_{\mathrm{d}}}}$ and $\left|k_{x}\right|>k_{0}$ will propagate quasiadiabatically from positions inside the transition area towards both the $-x$ and $+x$ directions as SPPs. In the homogeneous regions, which are outside the transition area, only a magnetic field with $k_{\mathrm{SPP}-x(+x)}=$

$k_{0} \sqrt{\frac{\varepsilon_{\mathrm{m}, \min (\max ) \varepsilon_{0}}}{\varepsilon_{\mathrm{m}, \min (\max )}+\varepsilon_{0}}}$ exists.

Due to the reversibility of light, we expect mutual conversion of SPPs and free-space propagating light. The dipole model we are using is reversible and can thus be used to explain coupling in both directions.

Generally, free-space propagating waves and surface waves can be coupled by a GNM, and the coupling can be enhanced if the material permittivity variation is suitably designed. In contrast, given uniform permittivity $\varepsilon_{\mathrm{m}}$, a free-space propagating wave cannot generate an SPP and a propagating SPP does not have a radiative component because of the homogeneity of the dipoles. With the tunability of nonconventional plasmonic materials, this new approach of coupling freespace light to SPP modes becomes possible. In the following section, we provide an example of such coupling based on full wave simulations.

\section{RESULTS AND DISCUSSION}

\section{Simulation}

The excitation of an SPP by a free-space propagating wave is confirmed by numerical simulations performed on Comsol Multiphysics. We use the same simulation schematic and permittivity distribution described in Figure 1a. The width of the permittivity transition area is approximately $2 \mu \mathrm{m}$.

We illuminate the GNM from above $(z>0)$ using a TM-polarized Gaussian beam, with a $1-\mu \mathrm{m}$ free-space wavelength and a $3-\mu \mathrm{m}$ waist (the radius of the $1 / e$ of the peak intensity), propagating perpendicularly to the surface. Figure $2 \mathrm{a}$ shows the electric field norm of the incident Gaussian beam without the GNM. For the above GNM example, Figure 2b shows the $z$ component of an electric field $\left(E_{z}\right)$. SPPs are clearly excited in the permittivity transition area and propagate along both the $+x$ and $-x$ directions. The $E_{z}$ distribution $1 \mathrm{~nm}$ above the GNM surface is plotted as a red curve in Figure 2c. On the $+x$ side, the period of the SPP is $0.71 \mu \mathrm{m}$, corresponding to the surface wave vector $k_{\mathrm{SP}+x}=k_{0} \sqrt{\frac{-2 \times 1}{-2+1}}=1.414 k_{0}$. On the $-x$ side, the period of the SPP is $0.95 \mu \mathrm{m}$, corresponding to the surface wave vector $k_{\mathrm{SPP}-x}=k_{0} \sqrt{\frac{-10 \times 1}{-10+1}}=1.054 k_{0}$. These correspondences indicate that 
a

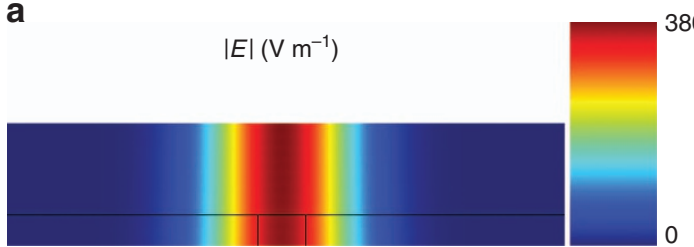

C

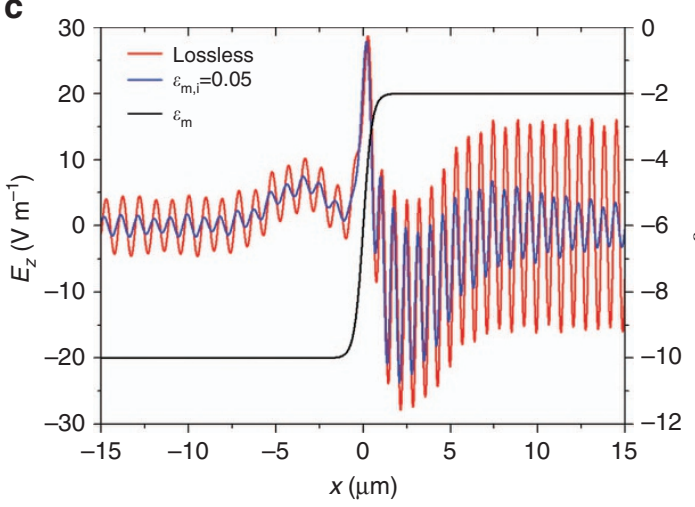

b
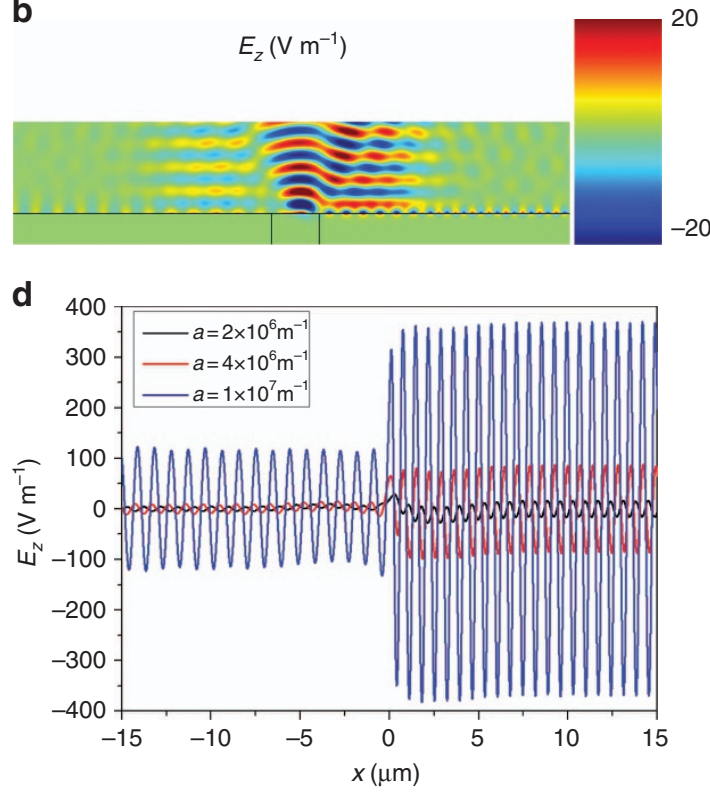

Figure 2 (a) Electric field magnitude of the incident Gaussian beam without a GNM. (b) $E_{Z}$ showing SPP excitation in both directions. (c) $E_{Z}$ distribution $1 \mathrm{~nm}$ above the interface (with and without loss) and corresponding permittivity distribution inside the GNM. (d) $E_{z}$ distribution with different permittivity changing rates $\alpha$ in the transition area.

when these GNM-generated SPPs propagate outside the transition area, they are similar to SPPs in homogeneous materials. For GNMs with very small loss $\varepsilon_{\mathrm{m}, i}=0.05$, the $E_{\mathrm{z}}$ distribution is shown as the blue curve in Figure 2c. The electric field decays from the transition area to both sides. The permittivity distribution of the GNM is also plotted in Figure $2 \mathrm{c}$ for identification purposes. By adjusting the changing rate $\alpha$ in the transition area and comparing the excited SPPs for different values of $\alpha$, as shown in Figure 2d, it is clear that the faster the permittivity changes in the transition area, the stronger the SPPs are excited, which is in agreement with our theory.

We investigated the coupling efficiency by integrating the power flow of the whole incident Gaussian beam and the excited SPPs. This comparison is similar to the method used in the literature ${ }^{34}$. Our coupling efficiency is lower but comparable to that of the widely used nanoslit method $(\sim 1 \%)$. The coupling efficiencies of simulations shown in Figure $2 \mathrm{~d}$ are $0.0007 \%, 0.04 \%$ and $0.2 \%$, respectively. If we reduce the waist of the incident Gaussian beam to $1.5 \mu \mathrm{m}$, which is still realistic, the efficiencies increase to $0.002 \%, 0.09 \%$ and $0.44 \%$, respectively. Unlike the higher efficiency end-fire coupling method, our method is nanofabrication free and may allow direct application due to the high compatibility with existing technologies.

We also emphasize that the new coupling mechanism in our design is not based on the adiabatic approximation. In an adiabatic system, no scattering/radiating components are present when an SPP is propagating along the surface. Reciprocity prevents the reversed coupling from free space to SPPs. Large adiabatic parameters $\left(\delta=\frac{\partial k^{-1}}{\partial x}\right)$ promote coupling from free space to SPP components inside transition layers, but limit excited SPP components from propagating out of the transition layers. As a reference, the maximum adiabatic parameters for the simulations in Figure $2 \mathrm{~d}$ are $0.04,0.08$ and 0.2 , respectively. The propagation of SPPs in transition layers is not adiabatic, but quasi-adiabatic, which allows for a simultaneous coupling process.

\section{Rainbow trapping}

This theory provides a guideline to many applications, including rainbow trapping ${ }^{35}$, nanofocusing ${ }^{36}$, or selectively local illumination ${ }^{37}$. Here, we present our practical design of 'rainbow trapping' with this theory. 'Rainbow trapping' has been demonstrated experimentally by metallic grating structures of various sizes. In a single 'rainbow trapping' nanostructure, multiple wavelengths over a broad spectral range are simultaneously slowed and trapped at different positions $s^{35,38}$. However, all current 'rainbow trapping' designs require external SPP generation or coupling methods, thereby increasing the complexity of the structure and causing higher dissipative losses during the additional propagation from the coupler to their primary nanostructures ${ }^{39,40}$. In our theory, 'rainbow trapping' can be realized on a gradient negative-permittivity planar surface without any nanostructures. Here, because doping control in semiconductors has been thoroughly studied and has become a reliable technique, we use the semiconductor doping method as an example to produce a gradient permittivity material for demonstration purposes. Similar to the schematic shown in Figure 1a, we may epitaxially deposit a semiinfinite indium antimonide (InSb) layer with a doping density of $n_{\text {epi }}=3 \times 10^{18} \mathrm{~cm}^{-3}$ on a semi-infinite InSb substrate with a doping density of $n_{\text {sub }}=1 \times 10^{18} \mathrm{~cm}^{-3}$. The actual local carrier density is not exactly the same as the dopant concentration, due to the diffusion of electrons. The thickness of the transition layer between the two InSb layers can be controlled during the epitaxial growth process by tuning the temperature of the dopant cell. The gradient-doped InSb is indeed a $n-n^{+}$homojunction, where free carriers (electrons) diffuse from the $n^{+}$side to the $n$ side until equilibrium is established, thereby forming a carrier concentration gradient ${ }^{41}$. After epitaxial growth, the InSb wafer may be cleaved to form an ultra-flat surface. Assuming homogeneity in the $y$ direction, the electron concentration $n(x)$ is only dependent on its $x$ coordinate. If the doping rate during the epitaxy process is carefully designed, then it is feasible to achieve an electron concentration distribution in the form of $n(x)=\frac{n_{\text {sub }}+n_{\text {epi }}}{2}+$ $\frac{n_{\text {epi }}-n_{\text {sub }}}{2} \tanh \left(1 \times 10^{6} x\right)$ to match the epsilon function, as previously 
defined. From its previous definition, the thickness of the transition layer is approximately $4 \mu \mathrm{m}$.

The permittivity in semiconductors can be described by the Drude model $^{42}$ as follows:

$$
\varepsilon_{\mathrm{InSb}}(\omega, x)=\varepsilon_{\infty-\mathrm{InSb}}-\frac{\omega_{\mathrm{p}}^{2}(x)}{\omega^{2}+i \omega \gamma}
$$

where the high frequency dielectric constant $\varepsilon_{\infty-\text { InSb }}=15.68$ and $\gamma=$ $1.79 \times 10^{12} \mathrm{rad} \mathrm{s}^{-1}$ is the collision frequency of electrons ${ }^{43}$. The expression $\omega_{\mathrm{p}}(x)=\sqrt{\frac{n(x) e^{2}}{m^{*} \varepsilon_{0}}}\left(\mathrm{rad} \mathrm{s}^{-1}\right)$ describes the plasma frequency of InSb, where $e$ is the electric charge, and $m^{*}$ is the effective mass of the electron (which is equal to 0.014 of the electron mass ${ }^{44}$ ).

In our simulation model, the plasma frequency $\omega_{\mathrm{p}}$ changes with the electron concentration variation along the $x$ direction. Taking into account Equation (3), we plot a series of 'local' dispersion curves of SPPs at different $x$ positions using the conventional SPP equation $k_{\mathrm{SPP}}(x)=k_{0} \sqrt{\frac{\varepsilon_{\mathrm{m}}(x) \varepsilon_{\mathrm{d}}}{\varepsilon_{\mathrm{m}}(x)+\varepsilon_{\mathrm{d}}}}$ shown in Figure 3a (for simplicity of demonstration, we removed the imaginary part of $\varepsilon_{\mathrm{InSb}}$ in the above results). Thus, different surface plasmon resonances are located at different $x$ positions.

Similar to our previous model, we simulated SPP generation and propagation by illuminating a Gaussian beam with a $30-\mu \mathrm{m}$ waist size at normal incidence on the InSb sample. The center of the incident Gaussian beam is aligned to the center of the transition area.

If the illumination frequency is below $f=18.3 \mathrm{THz}\left(\omega=1.15 \times 10^{14}\right.$ $\mathrm{rad} \mathrm{s}^{-1}$ ), then there are only intersections with these dispersion curves at small $k_{x}$ values, as demonstrated by the $17.5 \mathrm{THz}\left(\omega=1.1 \times 10^{14}\right.$ $\operatorname{rad~} s^{-1}$ ) illumination case (black dashed line in Figure 3a). Thus, for such illumination, SPPs can be generated and propagate towards both the $-x$ and $+x$ directions with a suitable decay factor, as shown in Figure $3 \mathrm{~b}$ by plotting $E_{z}$.

If the illumination frequency is between $f=18.3$ and $f=32.2 \mathrm{THz}$ $\left(\omega=2.0 \times 10^{14} \mathrm{rad} \mathrm{s}^{-1}\right)$, as demonstrated by the $22 \mathrm{THz}$ example $\left(\omega=1.4 \times 10^{14} \mathrm{rad} \mathrm{s}^{-1}\right.$, red dashed line in Figure $\left.3 \mathrm{a}\right)$, then there exists a specified $x$ position $\left(x_{0}\right)$, at which $\varepsilon_{\mathrm{m}}=-\varepsilon_{\mathrm{d}}$ and the surface wave vector $k_{x}$ reaches its maximum point, corresponding to both the group velocity and phase velocity approaching zero. For all dispersion curves with $x<x_{0}$, there is no intersection with the red dotted line, i.e., no propagating SPP mode exists and the excited SPPs' components in the $-x$ direction stop at the $x_{0}$ position. However, considering the finite loss given by the collision frequency of electrons, the group velocity of SPPs cannot reach zero, but slows down to 0.028 times light speed. Even considering this phenomenon, the electric field magnitude is strongly enhanced and localized in the nanoscale at that position, as shown in Figure 3c. We can define an enhancement factor as the ratio between the peak intensity of the localized spot and the peak intensity of the incident Gaussian beam. For the $22 \mathrm{THz}$ beam incident on the InSb sample, the peak intensity is enhanced by $\sim 2000$ times. If the illumination beam consists of multiple incident wavelengths, then we may have multiple zero velocity points at different positions, as shown in Figure 3d. This situation is the same as rainbow trapping and can directly enable wavelength sorting applications. An ultracompact spectrometer may be realized based on this phenomenon.

In addition to the semiconductor doping method, tunable carrier concentration control techniques are being developed. Electrical gating and optical pumping methods have shown the ability to provide a

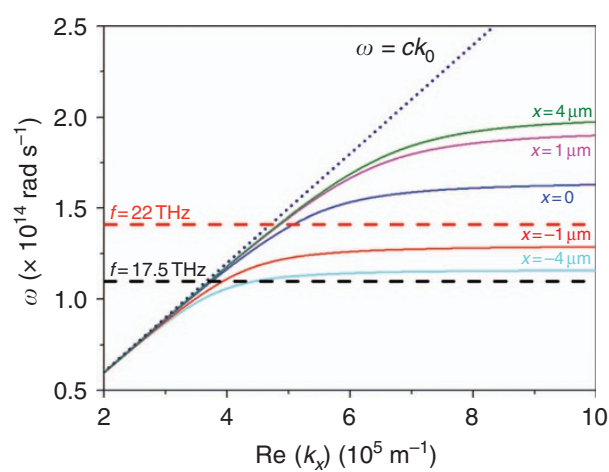

C

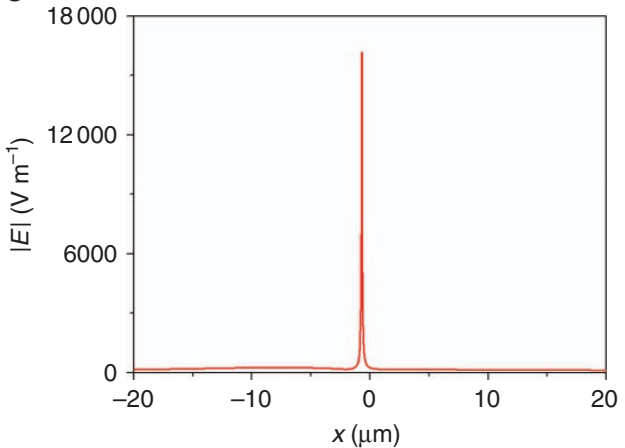

b

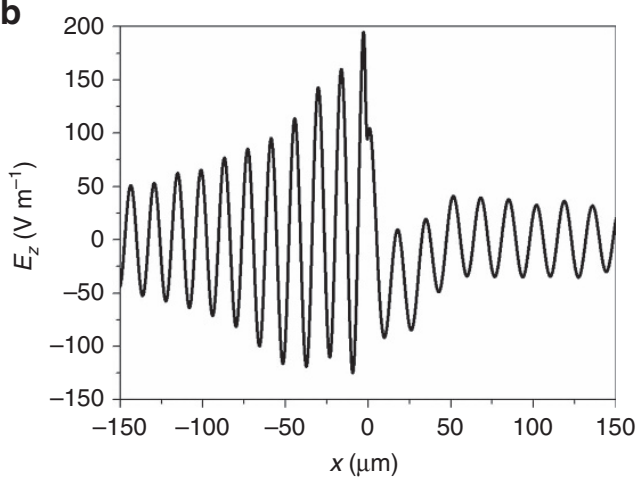

d

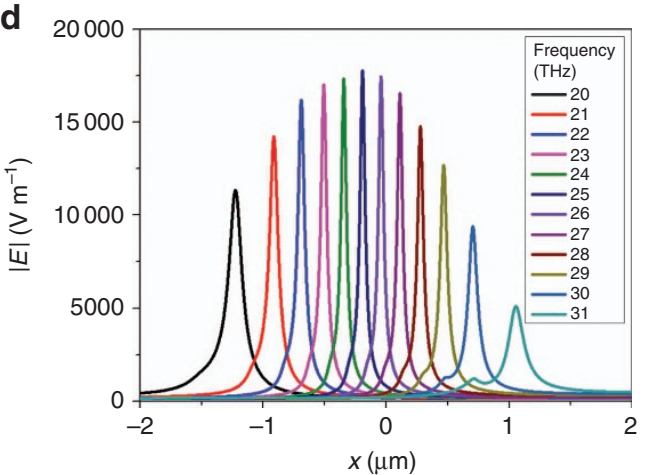

Figure 3 (a) Dispersion curves at different $x$ positions (for simplicity of demonstration, the imaginary part of $\varepsilon_{|n| n s}$ is removed). (b) $E_{z}$ distribution to highlight the excitation and propagation of SPPs along both the $-x$ and $+x$ directions under normal illumination with the incident frequency corresponding to the black dashed line (17.5 THz) in a. (c) electric field magnitude under normal illumination with the incident frequency corresponding to the red dashed line (22 THz) in a. (d) electric field magnitude along the interface under different frequency illuminations. 
tunable carrier concentration gradients. However, such gradients are typically ignored or treated as abrupt changes in the literature $e^{23,24,29}$. By taking advantage of the gradient, our method provides a new opportunity to enable plasmonic applications of existing electronic junctions.

\section{CONCLUSIONS}

In conclusion, we theoretically analyzed the excitation and propagation of SPPs on a planar interface between a homogeneous dielectric and a GNM. This theory provides a new approach for the design of plasmonic devices and a new light coupling method for planar optical devices and circuits, potentially enabled by non-conventional plasmonic materials. Because industrial-scale complementary metal oxidesemiconductor techniques provide precise control of the carrier distribution, the light couplers and concentrators predicted by this theory can potentially be integrated into an electronic-photonic system on a single chip ${ }^{45}$. This theory is also beneficial for the development of novel 'rainbow trapping' devices, which can enable various potential applications, including ultracompact spectroscopy.

\section{ACKNOWLEDGEMENTS}

We thank Kaichen Dong for useful discussions. This work is supported by the Samsung Advanced Institute of Technology under the Grant 037361-003 and the Hellman Family Foundation.

1 Maier SA. Plasmonics: Fundamentals and Applications. New York: Springer; 2007

2 Homola J, Yee SS, Gauglitz G. Surface plasmon resonance sensors: review. Sens Actuat B: Chem 1999; 54: 3-15.

3 Chung T, Lee S-Y, Song EY, Chun HG, Lee B. Plasmonic nanostructures for nano-scale bio-sensing. Sensors 2011; 11: 10907-10929.

4 Zhang X, Liu ZW. Superlenses to overcome the diffraction limit. Nat Mater 2008; 7: 435-441.

5 Barnes WL, Dereux A, Ebbesen TW. Surface plasmon subwavelength optics. Nature 2003; 424: 824-830.

6 Ghaemi HF, Thio T, Grupp DE, Ebbesen TW, Lezec HJ. Surface plasmons enhance optical transmission through subwavelength holes. Phys Rev B 1998; 58: 6779-6782.

7 Luo Y, Chamanzar M, Apuzzo A, Salas-Montiel R, Nguyen KN et al. On-chip hybrid photonic-plasmonic light concentrator for nanofocusing in an integrated silicon photonics platform. Nano Lett 2015; 15: 849-856.

8 Tang L, Kocabas SE, Latif S, Okyay AK, Ly-Gagnon D-S et al. Nanometre-scale germanium photodetector enhanced by a near-infrared dipole antenna. Nat Photonics 2008; 2: 226-229.

9 Novotny L, Bian RX, Xie XS. Theory of nanometric optical tweezers. Phys Rev Lett 1997; 79: 645-648.

10 Ye HP, Wang HF, Yeo SP, Qiu CW. Finite-boundary bowtie aperture antenna for trapping nanoparticles. Electromagn Waves 2013; 136: 17-27.

11 Nahata A, Linke RA, Ishi T, Ohashi K. Enhanced nonlinear optical conversion from a periodically nanostructured metal film. Opt Lett 2003; 28: 423-425.

12 van Nieuwstadt JAH, Sandtke M, Harmsen RH, Segerink FB, Prangsma JC et al. Strong modification of the nonlinear optical response of metallic subwavelength hole arrays. Phys Rev Lett 2006; 97: 146102.

13 Stern EA. Plasma radiation by rough surfaces. Phys Rev Lett 1967; 19: 1321-1324.

14 Ritchie $\mathrm{RH}$, Wilems RE. Photon-plasmon interaction in a nonuniform electron gas. I. Phys Rev 1969; 178: 372-379.

15 Ritchie RH, Arakawa ET, Cowan JJ, Hamm RN. Surface-plasmon resonance effect in grating diffraction. Phys Rev Lett 1968; 21: 1530-1533.

16 Shi HF, Wang CT, Du CL, Luo XG, Dong XC et al. Beam manipulating by metallic nanoslits with variant widths. Opt Express 2005; 13: 6815-6820.

17 Soref RA, Bennett BR. Electrooptical effects in silicon. IEEE J Quant Electron 1987; 23: $123-129$.

18 West PR, Ishii S, Naik GV, Emani NK, Shalaev VM et al. Searching for better plasmonic materials. Laser Photon Rev 2010; 4: 795-808.
19 Naik GV, Liu JJ, Kildishev AV, Shalaev VM, Boltasseva A. Demonstration of Al: ZnO as a plasmonic component for near-infrared metamaterials. Proc Natl Acad Sci USA 2012; 109: 8834-8838.

20 Cada M, Blazek D, Pistora J, Postava K, Siroky P. Theoretical and experimental study of plasmonic effects in heavily doped gallium arsenide and indium phosphide. Opt Mater Express 2015; 5: 340-352.

21 Naik GV, Boltasseva A. Semiconductors for plasmonics and metamaterials. Phys Status Solidi Rapid Res Lett 2010; 4: 295-297.

22 Park J, Kang J-H, Liu XG, Brongersma ML. Electrically tunable epsilon-near-zero (ENZ) metafilm absorbers. Sci Rep 2015; 5: 15754.

23 Liu YP, Tom K, Wang X, Huang CM, Yuan HT et al. Dynamic control of optical response in layered metal chalcogenide nanoplates. Nano Lett 2016; 16: 488-496.

24 Feigenbaum E, Diest K, Atwater HA. Unity-order index change in transparent conducting oxides at visible frequencies. Nano Lett 2010; 10: 2111-2116.

25 Tanaka H, Kamogawa M, Ohtsuki Y-H. The interaction between bulk plasmons and electromagnetic waves assisted by surface roughness. Proc Jpn Acad Ser B Phys Bio Sci 1999; 75: 190-194.

26 Kretschmann E. Scattering of light at rough surfaces due to the excitation of surface plasmons. Zeitschr Phys 1969; 227: 412-426.

27 Cho AY, Arthur JR. Molecular beam epitaxy. Prog Solid State Chem 1975; 10: 157-191.

28 Steinbusch TP, Tyagi HK, Schaafsma MC, Georgiou G, Rivas JG. Active terahertz beam steering by photo-generated graded index gratings in thin semiconductor films. Opt Express 2014; 22: 26559-26571.

29 Georgiou G, Tyagi HK, Mulder P, Bauhuis GJ, Schermer JJ et al. Photo-generated THz antennas. Sci Rep 2014; 4: 3584.

30 Cai WS, Shalaev V. Optical Metamaterials: Fundamentals and Applications. Berlin: Springer; 2010. p1-200.

31 Raether H. Surface Plasmons on Smooth and Rough Surfaces and on Gratings, Vol. 111. Berlin: Springer; 1988.

32 Born M, Wolf E, Bhatia AB. Principles of Optics: Electromagnetic Theory of Propagation, Interference and Diffraction of Light. Cambridge: Cambridge University Press; 2002.

33 Rodríguez-Fortuño FJ, Marino G, Ginzburg P, O'Connor D, Martínez A et al. Near-field interference for the unidirectional excitation of electromagnetic guided modes. Science 2013; 340: 328-330.

34 Hu HF, Zeng X, Ji DX, Zhu L, Gan QQ. Efficient end-fire coupling of surface plasmons on flat metal surfaces for improved plasmonic Mach-Zehnder interferometer. J App/ Phys 2013; 113: 053101.

35 Gan QQ, Ding YJ, Bartoli FJ. "Rainbow" trapping and releasing at telecommunication wavelengths. Phys Rev Lett 2009; 102: 056801.

36 Gramotnev DK, Bozhevolnyi SI. Nanofocusing of electromagnetic radiation. Nat Photonics 2014; 8: 13-22.

37 Cho WJ, Kim Y, Kim JK. Ultrahigh-density array of silver nanoclusters for SERS substrate with high sensitivity and excellent reproducibility. ACS Nano 2012; 6: 249-255.

38 Gan QQ, Gao YK, Wagner K, Vezenov D, Ding YJ et al. Experimental verification of the rainbow trapping effect in adiabatic plasmonic gratings. Proc Natl Acad Sci USA 2011; 108: 5169-5173.

39 Gramotnev DK, Vogel MW, Stockman MI. Optimized nonadiabatic nanofocusing of plasmons by tapered metal rods. J Appl Phys 2008; 104: 034311.

40 Vogel MW, Gramotnev DK. Optimization of plasmon nano-focusing in tapered metal rods. J Nanophotonics 2008; 2: 021852.

41 van Zeghbroeck B. 2011. Principles of semiconductor devices. Available from: http:// ecee.colorado.edu/ bart/book/book/chapter3/ch3 8.htm (accessed on 24 April 2016).

42 Fox M. Optical Properties of Solids. Oxford: Oxford University Press; 2001.

43 Hanham SM, Fernández-Domínguez AI, Teng JH, Ang SS, Lim KP et al. Broadband terahertz plasmonic response of touching InSb disks. Adv Mater 2012; 24: OP226-OP230.

$44 \mathrm{Kim}$ Y-S, Hummer K, Kresse G. Accurate band structures and effective masses for $\operatorname{lnP}$, InAs, and InSb using hybrid functionals. Phys Rev B 2009; 80: 035203.

45 Sun C, Wade MT, Lee Y, Orcutt JS, Alloatti L et al. Single-chip microprocessor that communicates directly using light. Nature 2015; 528: 534-538.

cc) $($ T) This work is licensed under a Creative Commons Attributioncc. ${ }_{\mathrm{BY}} \mathrm{NC}$ ND NonCommercial-NoDerivs 4.0 International License. The images or other third party material in this article are included in the article's Creative Commons license, unless indicated otherwise in the credit line; if the material is not included under the Creative Commons license, users will need to obtain permission from the license holder to reproduce the material. To view a copy of this license, visit http://

creativecommons.org/licenses/by-nc-nd/4.0/

(C) The Author(s) 2016

Supplementary Information for this article can be found on the Light: Science \& Applications' website (http://www.nature.com/lsa). 